\title{
Initial data for general relativity containing a marginally outer trapped torus
}

\author{
S. Husa \\ Institut für Theoretische Physik \\ Universität Wien \\ Boltzmanngasse 5, A-1090 Wien, Austria \\ e-mail: shusa@merlin.pap.univie.ac.at
}

(June 14, 1996)

\begin{abstract}
Asymptotically flat, time-symmetric, axially symmetric and conformally flat initial data for vacuum general relativity are studied numerically on $R^{3}$ with the interior of a standard torus cut out. By the choice of boundary condition the torus is marginally outer trapped, and thus a surface of minimal area. Apart from pure scaling the standard tori are parameterized by a radius $a \in[0,1]$, where $a=0$ corresponds to the limit where the boundary torus degenerates to a circle and $a=1$ to a torus that touches the axis of symmetry. Noting that these tori are the orbits of a $U(1) \times U(1)$ conformal isometry allows for a simple scheme to solve the constraint, involving numerical solution of only ordinary differential equations. Regular solutions can be constructed for $a \in] a_{0} \sim 0.14405,1[$. The tori are unstable minimal surfaces (i.e. only saddle points of the area functional) and thus can not be apparent horizons, but they are always surrounded by an apparent horizon of spherical topology, which is analyzed in the context of the hoop conjecture and isoperimetric inequality for black holes.
\end{abstract}

PACS numbers: 4.20.Ex, 2.40.Ky, 4.25.Dm

\section{INTRODUCTION}

The aim of this paper is to construct a class of asymptotically flat solutions to the vacuum constraint equations of general relativity that are somewhat unusual, namely they contain a marginally outer trapped torus. In the case of time symmetry discussed here, a marginally outer trapped surface has vanishing mean curvature and is a surface of minimal area (see Ref. [1] and references cited therein). The minimal tori are all unstable, that is, they are only saddle points of the area functional. In a recent discussion of minimal area tori and their connection with topological censorship Galloway [1] proved, that an unstable minimal torus locally can be deformed into an outer trapped surface. The outer boundary of the region containing trapped surfaces - the apparent horizon - is a stable marginally outer trapped surface of spherical topology (see Sec. V).

Motivation for this work comes from two sources: while the question of the existence of a regular solution to the constraints is of physical and mathematical significance in itself, analysis of given initial data with respect to the existence and properties of minimal surfaces and apparent horizons has received some interest in the discussion on cosmic censorship.

This work deals with the simplest situation where a toroidal minimal surface can be present, namely that of time-symmetry, axial symmetry and conformal flatness. The assumptions of time-symmetry and conformal flatness allow to use a conformal symmetry to turn the constraint from a partial differential equation (PDE) to a set of uncoupled ordinary differential equations (ODEs). Generalizations of the scheme that relax the restriction to conformal flatness and axial symmetry will be discussed below in Sec. VI.

The physical data are given by an asymptotically flat metric $\tilde{g}$ defined on a manifold $\tilde{M}$ that is taken to be $R^{3}$ with a region inside a torus $\partial \tilde{M}$ cut out. The physical metric $\tilde{g}$ is required to satisfy the vacuum Hamiltonian constraint of vanishing scalar curvature $R_{\tilde{g}}$ on $\tilde{M}$ plus the boundary condition of vanishing mean curvature $\tilde{p} / 2$ ( $\tilde{p}$ is the trace of the extrinsic curvature) at $\partial \tilde{M}$, making it a minimal surface:

$$
\begin{aligned}
R_{\tilde{g}}=0 & \text { on } \tilde{M}, \\
\tilde{p}=0 & \text { on } \partial \tilde{M} .
\end{aligned}
$$

The standard way to solve these equations is the conformal approach [2]. Restricting to conformal flatness we select the flat metric $\delta=d r^{2}+d z^{2}+r^{2} d \varphi^{2}$ on $\tilde{M}$ as our base metric and construct the physical metric as $\tilde{g}=\psi^{4} \delta$, which reduces Eq. (11) to an elliptic PDE for the conformal factor $\psi$.

For notational convenience we define the linear differential operators

$$
L_{g}:=-\triangle_{g}+\frac{1}{8} R_{g},
$$

the conformal Laplacian of a metric $g$, and

$$
B_{g}:=\frac{\partial}{\partial n}+\frac{1}{4} p
$$

where $\partial / \partial n$ is the normal derivative and $p$ is the trace of the extrinsic curvature (or twice the mean curvature) induced by the 3-metric $g$ on a hypersurface. Using the definitions of $L_{g}$ and $B_{g}$ the constraint equation (11) with boundary condition (2) turns into the problem of finding a conformal factor $\psi$, such that 


$$
\begin{aligned}
L_{\delta} \psi \equiv-\triangle_{\delta} \psi=0 & \text { on } \tilde{M} \\
B_{\delta} \psi=0 & \text { on } \partial \tilde{M}, \\
\psi>0, & \psi \rightarrow 1 \quad \text { as } \quad r^{2}+z^{2} \rightarrow \infty .
\end{aligned}
$$

We are left with the freedom to fix the boundary surface, taking it to be a standard torus in flat space, defined in cylindrical coordinates as a solution of

$$
z^{2}+(r-A)^{2}=a^{2} .
$$

Since a finite overall scaling of the coordinates bears no physical significance, we can fix $A=1$ for the boundary tori considered, which leaves us with the one parameter family $0 \leq a \leq 1$. The extremal case $a=0$ corresponds to a circle of unit radius in the $z=0$ plane, the other extreme, $a=1$, defines a torus which touches its axis of symmetry.

Using the method described below it is possible to continue the data smoothly inside the minimal tori to some finite extent, assuming vacuum and conformal flatness inside, but in general this assumption will lead to a nodal surface, and thus to a curvature singularity, if one proceeds too far. The obvious question whether it is possible to get a regular interior and nonnegative energy density of the matter is not dealt with here.

Regular solutions to the constraint equation (5) with boundary conditions (6, 7) exist for $a \in] a_{0} \sim 0.14405,1[$ (all numerical results are rounded to the last digit), where the parameter $a$ labels the boundary tori as defined above in Eq. (8). In the limit $a \rightarrow a_{0}$ the mass blows up, and the Sobolev quotient (an extension of the definition of the Yamabe number to manifolds with boundary) vanishes. Also the apparent horizon moves outward to infinity and becomes spherical in the limit. This behavior is familiar from the case when the (usual) Yamabe number approaches zero [3]. If $a$ is further decreased, the Sobolev quotient becomes negative, the mass diverges to minus infinity as $a \nearrow a_{0}$, and the conformal factor develops a nodal surface. In the other extremal case, when $a \rightarrow 1$, the mass stays finite, but a curvature singularity develops at the center, where the toroidal minimal surface touches the axis of symmetry. In this last case the solution can be given explicitly.

The toroidal minimal surfaces are all unstable, and part of their spectrum of perturbations was computed.

The ADM mass can be cast into an explicitly positive form in a way similar to Brill's result 迎, additionally it can be bounded from below.

Note that the initial data discussed here are perfectly viable for evolution, since an apparent horizon boundary condition [5] can be applied at the topologically spherical apparent horizon that surrounds the boundary torus. The minimal torus could then be viewed as a model of a bulk of matter or gravitational radiation that has enough energy to confine all information about it inside of the horizon. This comment also holds in the case $a \rightarrow 1$, when a curvature singularity appears at the center.
By using conformal symmetries, the PDE can be decomposed into an infinite set of uncoupled linear ODEs that were solved numerically. A convenient cutoff of the infinite series yields a highly accurate solution for the constraint. The existence of solutions can be decided by solving a single linear ODE, together with the high accuracy obtained for the actual solution, this enables a secure grip on the two interesting limiting cases.

The key technical input is the utilization of a $U(1) \times$ $U(1)$ conformal isometry that leaves the boundary torus invariant. In order to benefit from the conformal isometry the conformal method is reformulated for a compact base manifold $M$, taken to be the one-point compactification of $\tilde{M}$, that is $S^{3}$ with the interior of a torus cut out. The conformal ansatz $\tilde{g}=G^{4} g$, with $g$ the standard metric on $S^{3}$, results in an elliptic PDE similar to (5),

$$
L_{g} G=4 \pi c \delta_{\Lambda} \quad \text { on } M,
$$

but with a distributional source term: $\delta_{\Lambda}$ is the Dirac delta distribution concentrated at the point $\Lambda$ on $M$, which corresponds to asymptotic infinity of $\tilde{M}$, and $c$ is a positive constant that can be chosen arbitrarily for overall scaling. $G$ is thus a Green function for $c^{-1} L_{g}$ with boundary condition

$$
B_{g} G=0 \quad \text { on } \partial M .
$$

The existence of a positive Green function $G$ is determined by the sign of $\lambda_{1}$, the first eigenvalue of the conformal Laplacian with the present boundary condition, or equivalently by the sign of the Sobolev quotient $Q(g)$, a conformal invariant defined as

$$
Q(g):=\inf _{\varphi} \frac{\int_{M}\left(|\nabla \varphi|^{2}+\frac{1}{8} R_{g} \varphi^{2}\right) d v+\frac{1}{4} \int_{\partial M} p \varphi^{2} d \sigma}{\left(\int_{M} \varphi^{6} d v\right)^{1 / 3}},
$$

which is a generalization of the Yamabe functional [6] (defined for manifolds without boundary) to manifolds with boundary [7] and has the same sign as $\lambda_{1}$.

It can be shown in a manner equivalent to the noboundary case dealt with in [8], that a positive $G$ exists iff $\lambda_{1}$ is positive. Furthermore, when $G>0$, the positive mass theorem holds for $\tilde{g}=G^{4} g$ [9].

The rest of this paper is organized as follows: In the next section (III) conformal compactification via inverse stereographic projection from $R^{3}$ to $S^{3}$ and toroidal coordinates thereon will be discussed. In Sec. III the method to solve the constraint numerically using toroidal coordinates will be developed, including numerical solution for the first eigenvalue of the conformal Laplacian. Section IV deals with the instability of the minimal tori by considering normal variations of the surfaces. Apparent horizons are located and analyzed with respect to the isoperimetric inequality for black holes [10] and the hoop conjecture [11] in Sec. V. A discussion of the results, with emphasis on the limiting cases, is given in section V1. 


\section{COMPACTIFICATION AND CONFORMAL SYMMETRY}

For every choice of boundary torus $\Sigma_{\gamma}$, that is a torus described by Eq. (8) with $A=1$ and parameterized by $\gamma:=\sqrt{1-a^{2}} \leq 1$, we define a conformal rescaling from the flat metric $\delta$ on $R^{3}$ to a metric $g(\gamma)$ defined on $S^{3}$ by

$$
g(\gamma)=\Omega(r, z ; \gamma)^{2} \delta, \quad \Omega(r, z ; \gamma)=\frac{2}{\gamma^{2}+r^{2}+z^{2}}
$$

which corresponds to an inverse stereographic projection. The notation $f(\bar{r}, \bar{z} ; \gamma)$ is chosen to denote dependence of a function $f$ on both the spatial coordinates and the parameter $\gamma$, frequently this will be shortened to writing $f(\gamma)$. With the chosen normalization $g(\gamma)$ is the standard metric on the 3 -sphere of radius $1 / \gamma$, which is conveniently written as

$$
g(\gamma)=\frac{d \bar{r}^{2}}{1-\gamma^{2} \bar{r}^{2}}+\left(1-\gamma^{2} \bar{r}^{2}\right) d \bar{z}^{2}+\bar{r}^{2} d \varphi^{2}
$$

in toroidal coordinates $(\bar{r}, \bar{z}, \varphi)$ taking ranges $0 \leq \bar{r} \leq$ $1 / \gamma, 0 \leq \gamma \bar{z}, \varphi \leq 2 \pi$. A discussion of the construction of this coordinate system on $S^{3}$ of unit radius is given in [12]. The coordinate $\bar{r}$ as a function on the $(r, z)$-half plane can be read off from Eqs. (12) and (13) yielding

$$
\bar{r}(r, z ; \gamma)=\Omega(r, z ; \gamma) r
$$

The relation between the coordinate $\bar{z}$ and the original cylindrical coordinates is

$$
\cos (\gamma \bar{z})=\frac{1-\gamma^{2} \Omega(r, z ; \gamma)}{\sqrt{1-\gamma^{2} \bar{r}(r, z ; \gamma)^{2}}}, \quad \operatorname{sign}(\bar{z})=\operatorname{sign}(z)
$$

The point $\Lambda$ on $S^{3}$, corresponding to asymptotic infinity of $R^{3}$, is given by $\bar{r}=\bar{z}=0$.

The vector fields $(\partial / \partial \varphi)^{a},(\partial / \partial \bar{z})^{a}$ form a pair of commuting, orthogonal and hypersurface-orthogonal Killing vector fields of $g(\gamma)$ spanning the surfaces of constant $\bar{r}$. For $\bar{r} \neq 0,1 / \gamma$ these are flat tori of constant mean curvature changing sign at the so called Clifford torus for which $\bar{r}=1 / \gamma \sqrt{2}$. Going back to the flat metric and cylindrical coordinates, a torus $\bar{r}=$ const. is mapped to a standard torus as defined by Eq. (8) with parameters $A=1 / \bar{r}, a=\sqrt{1-\gamma^{2} \bar{r}^{2}} / \bar{r}$, in particular the torus $\bar{r}=1$ is mapped to the boundary torus $\Sigma_{\gamma}$. The sets $\bar{r}=0$ (respectively $\bar{r}=1 / \gamma$ ) are linked great circles on $S^{3}$, corresponding to the $z$-axis (respectively the circle $z=0, r=\gamma$ ) after stereographic projection.

The totally geodesic surfaces $\bar{z}=$ const. $(\varphi=$ const. $)$ are metric hemispheres intersecting at $\bar{r}=1 / \gamma(\bar{r}=0)$ with $\{\gamma \bar{z}=\alpha\} \cup\{\gamma \bar{z}=\alpha+\pi\}(\{\varphi=\beta\} \cup\{\varphi=\beta+\pi\})$ being smoothly embedded $S^{2}$ 's. Under stereographic projection, the surfaces $\gamma \bar{z}=$ const. do not change topology except for $\{\gamma \bar{z}=0\} \cup\{\gamma \bar{z}=\pi\}$ which gets decompactified into $\{z=0, r \geq \gamma\} \cup\{z=0, r \leq \gamma\}$, i.e. the equatorial plane $z=0$. In flat space $\{\gamma \bar{z}=\alpha\} \cup\{\gamma \bar{z}=\alpha+\pi\}$ is a sphere centered at $r=0, z=\gamma \cot \gamma \bar{z}$ with radius $\sqrt{\gamma^{2}\left(\cot ^{2} \gamma \bar{z}-1\right)+2}$.

Note that for $\gamma=0$ the radius of $S^{3}$ becomes infinite, corresponding to $R^{3}$, and the coordinates $\bar{r}, \bar{z}$ become inverted cylindrical coordinates thereon:

$$
\bar{r}=\frac{2 r}{r^{2}+z^{2}}, \quad \bar{z}=\frac{2 z}{r^{2}+z^{2}} .
$$

The tori $\bar{r}=$ const., corresponding to $A=a=1 / \bar{r}$, all touch the $z$-axis in this limiting case.

We have thus constructed a family of foliations of $R^{3}$ by standard tori, with each element defined by selection of a boundary torus $\Sigma_{\gamma}$ that is contained in the foliation. For every foliation labeled by $\gamma$ we defined in Eq. (12) a conformal rescaling with compactifying factor $\Omega(r, z ; \gamma)$. Since $\Omega$ is independent of $\varphi,(\partial / \partial \varphi)^{a}$ is a Killing vector of $g$, but $(\partial / \partial \bar{z})^{a}$ is only a conformal Killing vector of $g$.

The reason for the chosen scaling of $S^{3}$ as opposed to working on $S^{3}$ of fixed radius can bee seen when rescaling to the standard metric $\hat{g}$ on $S^{3}$ of unit radius (with the notation of [12]):

$$
\hat{g}:=\gamma^{2} g(\gamma)=\frac{d \rho^{2}}{1-\rho^{2}}+\left(1-\rho^{2}\right) d \chi^{2}+\rho^{2} d \varphi^{2}
$$

using rescaled toroidal coordinates $(\rho, \chi, \varphi)$

$$
\rho(r, z ; \gamma):=\gamma \bar{r}(r, z ; \gamma), \quad \chi(r, z ; \gamma):=\gamma \bar{z}
$$

taking ranges $0 \leq \rho \leq 1,0 \leq \chi, \varphi \leq 2 \pi$. The boundary torus $\Sigma_{\gamma}$ that was defined by $\bar{r}=1$ before is now specified by $\rho=\gamma$, so that the region of physical interest, $0 \leq \rho \leq$ $\gamma$ shrinks to zero volume in the limit $\gamma \rightarrow 0$. The problem of finding a conformal factor solving the rescaled equation $L_{\hat{g}} \hat{G}=4 \pi \sqrt{2} \delta_{\Lambda}$ then becomes ill-defined, the conformal factor $\hat{G}$ (and thus the mass of $\hat{G}^{4} \hat{g}$ ) blows up for $\gamma \rightarrow 0$.

In order to formulate the constraint equation explicitly we need to write down the operators $L_{g}$ and $B_{g}$ in our toroidal coordinate system. The extrinsic curvature of a torus of constant $\bar{r}$ with respect to the metric $g_{a b}$ is (the semicolon denotes the covariant derivative as usual)

$$
p_{a b}=\bar{r} \sqrt{1-\gamma^{2} \bar{r}^{2}}\left(\varphi_{; a} \varphi_{; b}-\gamma^{2} \bar{z}_{; a} \bar{z}_{; b}\right) .
$$

At $\partial M(\bar{r}=1)$ this yields

$$
p=p_{a}^{a}=\frac{1-2 \gamma^{2}}{\sqrt{1-\gamma^{2}}}
$$

which vanishes at the Clifford torus given by $\gamma_{c}=1 / \sqrt{2}$, where $\partial M$ is maximal. The extrinsic curvature $\tilde{p}_{a b}$ with respect to the physical metric $\tilde{g}$ is

$$
\tilde{p}_{a b}=G^{2}\left(p_{a b}+2 g_{a b} n^{c} G_{; c}\right),
$$

where $n^{c}$ is the unit normal vector field on $\partial M$. Note that since $p_{a b}$ has Lorentz signature, in particular it is 
not proportional to $g$ (14), by the conformal rescaling formula for the extrinsic curvature (16) it is not possible to get $\tilde{p}_{a b}=0$ by a conformal transformation. As a consequence the minimal tori cannot be stable, since in that case they would have to be totally geodesic $\left(\tilde{p}_{a b}=0\right)$ - (see [1]).

Finally we can write down the operators $L_{\gamma}:=L_{g_{\gamma}}$ and $B_{\gamma}:=B_{g_{\gamma}}-$ simplifying their labels - defined in Eqs. (9, 10) explicitly:

$$
\begin{aligned}
B_{\gamma} & =\sqrt{1-\gamma^{2}}\left(\frac{\partial}{\partial \bar{r}}+\frac{1-2 \gamma^{2}}{4 \sqrt{1-\gamma^{2}}}\right), \\
L_{\gamma}= & -\left(1-\gamma^{2} \bar{r}^{2}\right) \frac{\partial^{2}}{\partial \bar{r}^{2}}-\frac{1-3 \gamma^{2} \bar{r}^{2}}{\bar{r}} \frac{\partial}{\partial \bar{r}} \\
& -\frac{1}{1-\gamma^{2} \bar{r}^{2}} \frac{\partial^{2}}{\partial \bar{z}^{2}}-\frac{1}{\bar{r}^{2}} \frac{\partial^{2}}{\partial \varphi^{2}}+\frac{3}{4} \gamma^{2} .
\end{aligned}
$$

\section{SOLUTION OF THE CONSTRAINT EQUATION}

\section{A. Formulation}

From the choices made above our physical data are determined by the single parameter $\gamma$, which labels the position of the boundary torus. This defines the physical metric $\tilde{g}(\gamma)=G(\gamma)^{4} g(\gamma)$, where $G(\gamma)$ satisfies the equations

$$
\begin{aligned}
& L_{\gamma} G(\gamma)=4 \pi \sqrt{2} \delta_{\Lambda} \quad \text { on } M \\
& B_{\gamma} G(\gamma)=0 \quad \text { on } \partial M
\end{aligned}
$$

with operators $L_{\gamma}$ and $B_{\gamma}$ given by the expressions $(17,18)$, the boundary torus is located at the fixed coordinate value $\bar{r}=1$ and the scale factor is chosen as $c=\sqrt{2}$. The rescaling to flat space defined in Eq. (12) corresponds to a Green function $G_{0}$,

$$
G_{0}(\gamma)=\Omega(\gamma)^{-1 / 2}=\gamma\left(1-\sqrt{1-\gamma^{2} \bar{r}^{2}} \cos \gamma \bar{z}\right)^{-1 / 2}
$$

where $G_{0}(\gamma)$ satisfies Eq. (19) but with the boundary condition (20) replaced by regularity on $S^{3} \backslash\{\Lambda\}$. The asymptotic behavior of $G$ near $\Lambda$ is known [8] to be

$$
G=G_{0}+\frac{m}{2 \sqrt{2}}+O\left(G_{0}^{-1}\right)
$$

where $m$ is the ADM-mass of the physical metric $\tilde{g}$. By the positive mass theorem [13 $m$ is positive provided $\lambda_{1}$ is positive.

The relation to the original formulation of the constraint problem in Eqs. (5-7) is as follows:

$$
\tilde{g}=G^{4} g=\psi^{4} \delta=\psi^{4} \Omega^{-2} g \Rightarrow \psi=\Omega^{-1 / 2} G,
$$

$$
\psi=1+\frac{m}{2 \sqrt{r^{2}+z^{2}}}+O(\Omega) .
$$

The limiting cases $\gamma=0,1$ can be considered as singular situations, and the question arises how this will effect the physical metric $\tilde{g}(\gamma)$, in particular its existence. For $\gamma<\gamma_{c}$ we have $p$ positive (see Eq. (15)) whence is the Sobolev quotient $Q(g)$ defined in Eq. (11) - a positive Green function thus exists and the mass of the physical metric $\tilde{g}(\gamma)$ is positive. On the other hand $p$ is negative for $\gamma>\gamma_{c}$, so that a change of sign becomes possible for $Q(g)$ at some $\gamma, \gamma_{c}<\gamma<1$. This indeed happens at the numerically calculated value $\gamma_{0}=0.98957$, corresponding to $a=0.14405$. As one expects, this case is analogous to the Yamabe number going to zero in the usually considered case of manifolds without boundary. The mass diverges to plus infinity as $\gamma$ approaches $\gamma_{0}$ from below, and to minus infinity when taking the limit from above.

For $\gamma \rightarrow 0$ the metric $g$ becomes the flat metric on the infinite cylinder $0 \leq \bar{r} \leq 1,-\infty<\bar{z}<\infty, 0 \leq \varphi \leq 2 \pi$ :

$$
g(0)=d \bar{r}^{2}+d \bar{z}^{2}+\bar{r}^{2} d \varphi^{2}
$$

The point $\Lambda$, representing physical infinity, is at $\bar{r}=\bar{z}=$ 0 , the unphysical infinity, $\bar{z}= \pm \infty$ corresponds to the physical center $r=z=0$. The constraint Eq. (9) reduces to the cylindrically symmetric Poisson equation for a point source

$$
L_{0} G(0)=-\left(\frac{\partial^{2}}{\partial \bar{r}^{2}}+\frac{1}{\bar{r}} \frac{\partial}{\partial \bar{r}}+\frac{\partial^{2}}{\partial \bar{z}^{2}}\right) G(0)=4 \pi \sqrt{2} \delta_{\Lambda},
$$

with boundary condition

$$
\left.\left(\frac{\partial}{\partial \bar{r}}+\frac{1}{4}\right) G(0)\right|_{\bar{r}=1}=0 .
$$

The explicit limiting solution is derived in Appendix A as

$$
\begin{aligned}
& \frac{1}{\sqrt{2}} G(\bar{r}, \bar{z} ; 0)=\frac{1}{\sqrt{\bar{r}^{2}+\bar{z}^{2}}} \\
& \quad+\frac{2}{\pi} \int_{0}^{\infty} \cos (\omega \bar{z}) \frac{4 \omega\left(K_{1}(\omega)-K_{0}(\omega)\right)}{4 \omega I_{1}(\omega)+I_{0}(\omega)} I_{0}(\omega \bar{r}) d \omega .
\end{aligned}
$$

As is discussed in Appendix A, $G(\bar{r}, \bar{z} ; 0)$ is a regular positive function for $0 \leq \bar{r} \leq 1,-\infty<\bar{z}<\infty, \bar{r}^{2}+\bar{z}^{2}>$ 0 . The ADM-mass of $\tilde{g}(0)=G(0)^{4} g(0)$ is $m=3.877$, in particular it is finite. For $|\bar{z}|$ large $G$ decays exponentially,

$$
G(\bar{r}, \bar{z} ; 0) \propto \exp (-\alpha|\bar{z}|), \quad \alpha=0.6856
$$

it is thus zero at the physical origin $r=z=0$. There the Kretschmann invariant diverges as $\tilde{R}_{a b c d} \tilde{R}^{a b c d}[\tilde{g}(0)] \propto$ $\exp (8 \alpha|\bar{z}|)$, the physical metric thus has a curvature singularity at the origin, where the minimal torus touches the axis of symmetry. 


\section{B. Existence of Solutions}

As stated above, the existence of a solution to the constraint, that is a positive Green function for the elliptic operator $L_{\gamma}$ with boundary condition given by Eq. (10), is equivalent to positivity of the lowest eigenvalue $\lambda_{1}$ of $L_{\gamma}$. Calling the eigenfunctions $\theta$ we obtain the eigenvalue problem

$$
L_{\gamma} \theta=\lambda \theta,\left.\quad B_{\gamma} \theta\right|_{\bar{r}=1}=0,
$$

which is separable and the solutions can be written as

$$
\theta_{\lambda m n}(\bar{r}, \varphi, \bar{z})=\vartheta_{\lambda m n}(\bar{r}) \sin (n \varphi+\alpha) \sin (m \gamma \bar{z}+\beta),
$$

where $\alpha$ and $\beta$ are arbitrary angles and we are left to solve an equation for $\vartheta$,

$$
\left(L_{\bar{r} \gamma}+\frac{m^{2}}{1-\gamma^{2} \bar{r}^{2}}+\frac{n^{2}}{\bar{r}^{2}}\right) \vartheta_{\lambda m n}=\lambda \vartheta_{\lambda m n}
$$

where $L_{\bar{r} \gamma}$ is the 'radial' part of the conformal Laplacian $L_{\gamma}$,

$$
L_{\bar{r} \gamma}=-\left(1-\gamma^{2} \bar{r}^{2}\right) \frac{\partial^{2}}{\partial \bar{r}^{2}}-\frac{1-3 \gamma^{2} \bar{r}^{2}}{\bar{r}} \frac{\partial}{\partial \bar{r}}+\frac{3}{4} \gamma^{2} .
$$

Since the first eigenfunction is non-degenerate [14 and has therefore to depend trivially on the angular coordinates $\varphi$ and $\bar{z}$, we may set $m=n=0$ when looking for the lowest eigenvalue $\lambda_{1}$ :

$$
L_{\bar{r} \gamma} \vartheta_{\lambda 00}=\lambda \vartheta_{\lambda 00}
$$

The existence question has thus been reduced to an ODE of Sturm-Liouville type which can be solved by standard methods. In this case routine d02kef from the NAG Fortran Library [15] was chosen to calculate eigenvalues and eigenfunctions.

The lowest eigenvalue is found to be positive for $\gamma<$ $\gamma_{0}=0.98957$, and negative for $\gamma$ greater, see figure (11) and table (I). For $\gamma=0$ Eq. 25) reduces to Bessel's equation:

$$
\bar{r}^{2} \vartheta^{\prime \prime}+\bar{r} \vartheta^{\prime}+\lambda \bar{r}^{2} \vartheta=0, \quad \vartheta^{\prime}+\left.\frac{1}{4} \vartheta\right|_{\bar{r}=1}=0 .
$$

The regular solution is (apart from arbitrary scaling) $\vartheta=$ $J_{0}(\sqrt{\lambda} \bar{r})\left(J_{0}\right.$ is the Bessel function of order 0$)$ where $\lambda$ is chosen to satisfy the boundary condition

$$
J_{0}^{\prime}(\sqrt{\lambda} \bar{r})+\left.\frac{1}{4} J_{0}(\sqrt{\lambda} \bar{r})\right|_{\bar{r}=1}=0,
$$

which yields $\lambda_{1}=0.4700$.

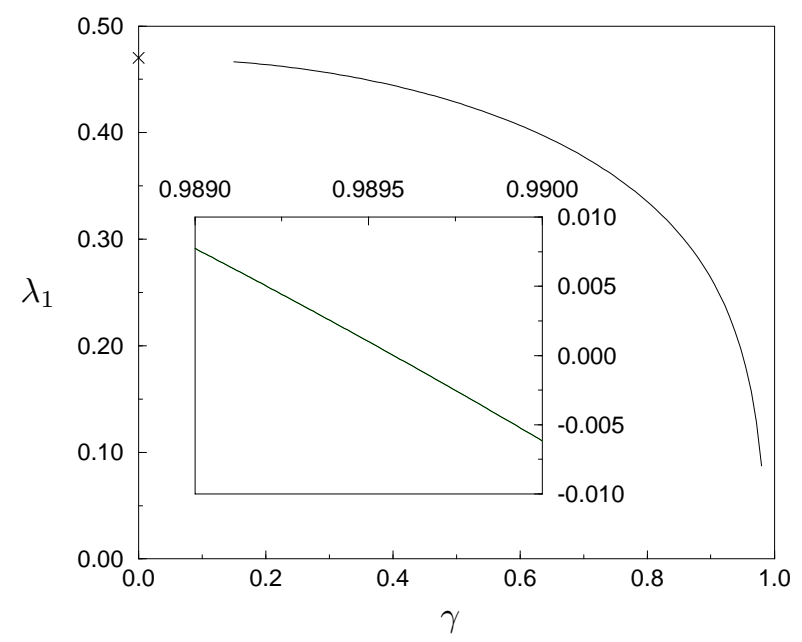

FIG. 1. The lowest eigenvalue $\lambda_{1}$ of the conformal Laplacian is plotted as a function of $\gamma$, the insert shows that $\lambda_{1}$ gets negative for $\gamma>\gamma_{0}$. The value for $\gamma=0$ is marked by an $\times$.

\section{Transforming the Constraint to ODEs}

Since the singular behavior of $G(\bar{r}, \bar{z} ; \gamma)$ at $\Lambda$ makes a direct numerical solution for the Green function inconvenient, the first step in solving the system (9, 10) is to regularize equation (9). We split $G$ into the singular part $G_{0}$ and a regular part $\phi$ by

$$
G(\gamma)=G_{0}(\gamma)+\phi(\gamma)
$$

The asymptotic behavior of $\phi$ near $\Lambda$ is

$$
\phi=\frac{m}{2 \sqrt{2}}+O\left(G_{0}^{-1}\right)
$$

from the asymptotic behavior of the Green function (21), $m$ is the ADM-mass of the physical metric $\tilde{g}$. Calculation of the mass is thus as simple as evaluating the numerical solution $\phi$ at the point $\Lambda$. Insertion of (26) into (9, 10) yields an elliptic problem for $\phi$ :

$$
\begin{aligned}
L_{\gamma} \phi & =0 \\
\left.B_{\gamma} \phi\right|_{\bar{r}=1} & =-\left.B_{\gamma} G_{0}\right|_{\bar{r}=1} \\
& =\left.\frac{G_{0}}{2}\left(\frac{\cos \gamma \bar{z} G_{0}^{2}}{\sqrt{1-\gamma^{2}}}-\frac{1-2 \gamma^{2}}{2\left(1-\gamma^{2}\right)}\right)\right|_{\bar{r}=1}=: b(\bar{z}, \gamma) .
\end{aligned}
$$

Note that the boundary condition becomes singular for $\gamma \rightarrow 1$.

The above partial differential equation in two dimensions can be turned into a system of a countable set of uncoupled ODEs by decomposing $\phi$ and $b$ into a cosine series (the sine terms of the Fourier decomposition are zero due to the symmetry with respect to reflection at the equator): 


$$
\phi=\sum_{n=0}^{\infty} \phi_{n}(\bar{r}) \cos (n \gamma \bar{z}), \quad b=\sum_{n=0}^{\infty} b_{n}(\bar{r}) \cos (n \gamma \bar{z}) .
$$

This gives a boundary value ODE problem for every $n$ (prime denoting differentiation with respect to $\bar{r}$ ):

$$
\begin{aligned}
\left(L_{\bar{r} \gamma}-\frac{n^{2}}{1-\gamma^{2} \bar{r}^{2}}\right) \phi_{n} & =0 \\
\left.\left(\phi_{n}^{\prime}+\frac{1-2 \gamma^{2}}{4\left(1-\gamma^{2}\right)} \phi_{n}\right)\right|_{\bar{r}=1} & =b_{n}(\gamma), \\
\left.\phi_{n}^{\prime}\right|_{\bar{r}=0} & =0 .
\end{aligned}
$$

The last condition ensures regularity on $\bar{r}=0$, the $z$-axis. By integrating the ODEs beyond $\bar{r}=1$ it is possible to continue the solution smoothly inside the minimal tori to some finite extent, but this will eventually lead to a nodal surface, and thus to a curvature singularity.

Note that, for $n=0$, Eq. (28) corresponds to Eq. (25), and correspondingly only $\phi_{0}$ blows up for $\gamma_{0}$, the higher order terms pass through $\lambda_{1}=0$ smoothly.

The equations are solved by a shooting and matching method (NAG routine d02agf [15]) for $n=1, \ldots, N$, where $N$ is suitably chosen to make the truncation error for $\varphi$ reasonably small.

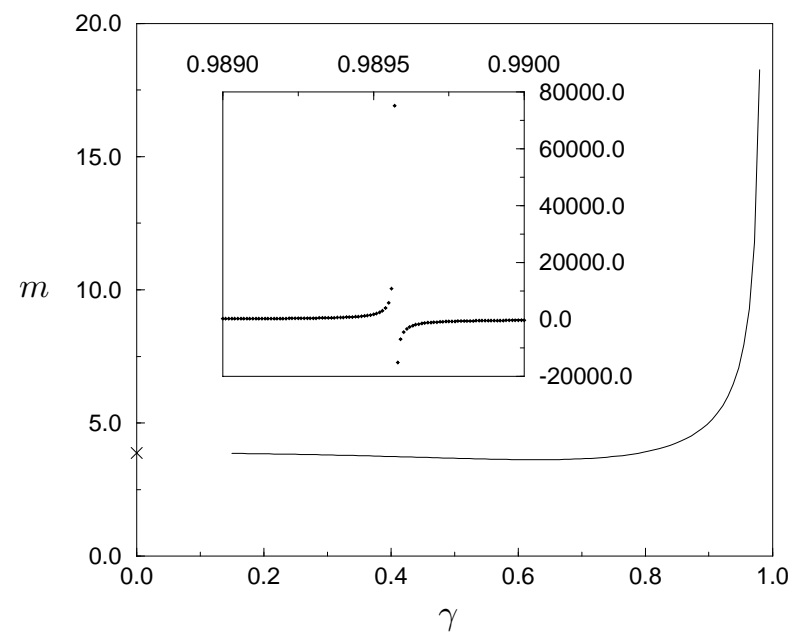

FIG. 2. The ADM mass is plotted as a function of $\gamma$, the insert shows the pole of $m$ at $\gamma=\gamma_{0}$. The value for $\gamma=0$ is marked by an $\times$.

\section{Tests of Numerical Accuracy}

For every approximate solution

$$
\phi_{N}=\sum_{n=0}^{N} \phi_{n}(\bar{r}) \cos (n \gamma \bar{z})
$$

with cutoff-parameter $N$ one may ask how well the Hamiltonian constraint is satisfied. Due to numerical inaccuracies and cutting off the Fourier series at finite $N$, Eqs. (5) and (5) will not be satisfied identically, but instead there will appear two functions $E_{1}$ and $E_{2}$ such that

$$
\triangle_{\delta} \psi=E_{1} \not \equiv 0, \quad B_{\delta} \psi=E_{2} \not \equiv 0
$$

where $E_{1}, E_{2}$ should be small in a sense that has yet to be determined. From the Fourier series expansion it can be seen, that increasing $N$ will increase $\left|E_{1}\right|$ too, since the conformal Laplacian annihilates every single term in the above Fourier series, $L_{g} \phi_{n} \cos (n \gamma \bar{z})=0$, for $\phi_{n}$ known exactly. $E_{1}$ is thus simply the sum of all numerical inaccuracies of the individual Fourier terms, and the sole purpose of increasing $N$ is to yield better satisfaction of the boundary conditions, that is to decrease $E_{2}$.

Let us deal with the smallness of $E_{1}$ first. In the case of a vacuum spacetime, where the scalar curvature has to vanish, there does not exist an a priori measure for $E_{1}$. Following [16] the error can be written as $\tilde{R}=16 \pi \rho_{\text {res }}$, where $\rho_{\text {res }}$ can be interpreted as the mass-energy density of matter for a non-vacuum spacetime. The criterion of accuracy derived from this viewpoint is the smallness of the total amount of mass represented by $\rho_{\text {res }}$ as compared to the ADM-mass.

The residual of the Hamiltonian constraint is defined as

$$
\rho_{\text {res }}=\frac{8}{16 \pi} \psi^{-5} L_{\delta} \psi
$$

yielding the residual mass

$$
m_{r e s}=\int_{V}\left|\rho_{r e s}\right| d V=\int_{V}\left|\rho_{r e s}\right| \psi^{6} d^{3} x .
$$

Since the solution has been constructed in toroidal coordinates, one gets better results, if derivatives are computed with respect to these, so $m_{\text {res }}$ is rather evaluated in the form

$$
m_{\text {res }}=\frac{1}{2 \pi} \int_{V} \psi \Omega^{-1 / 2}\left|L_{g} G\right| \bar{r} d \bar{r} d \bar{z} d \varphi .
$$

The volume of integration has to be chosen carefully. Since the domain of computation is infinite with respect to the physical metric, integration over the whole computational domain would yield an infinite result, the integration was thus restricted to $\sqrt{r^{2}+z^{2}} \leq 50 \mathrm{~m}$.

An alternative way to control the numerical error that is especially useful to control fulfillment of the boundary conditions can be constructed by putting the ADM mass into an explicitly positive form analogous to the expression derived for Brill-waves in [1]. From Eq. (5) we get ( $\partial$ is the flat derivative operator)

$$
0=\frac{\triangle_{\delta} \psi}{\psi}=\partial^{2} \log \psi+(\partial \log \psi)^{2}
$$


which can be integrated with respect to the flat background metric to give

$$
\int_{\partial M} \frac{n^{a} \partial_{a} \psi}{\psi} d A+\int_{M}\left(\frac{\partial \psi}{\psi}\right)^{2} d V=0
$$

The boundary $\partial M$ consists of spatial infinity and the boundary torus $\Sigma$, inserting the definition of the ADM mass $m$ yields

$$
m=\frac{1}{2 \pi}\left(\int_{\Sigma} \frac{n^{a} \partial_{a} \psi}{\psi} d A+\int_{M}\left(\frac{\partial \psi}{\psi}\right)^{2} d V\right) .
$$

Using the boundary condition $n^{a} \partial_{a} \psi+\psi p / 4=0$ allows to calculate the first integral explicitly as

$$
m_{\Sigma}:=\frac{1}{2 \pi} \int_{\Sigma} \frac{n^{a} \partial_{a} \psi}{\psi} d A=-\frac{1}{8 \pi} \int_{\Sigma} p d A=\frac{\pi}{2}
$$

resulting in

$$
m=\frac{\pi}{2}+\frac{1}{2 \pi} \int_{M}\left(\frac{\partial \psi}{\psi}\right)^{2} d V=: m_{\Sigma}+m_{V} .
$$

The minimal mass of the metrics in the sequence considered is thus bounded from below by $m>\pi / 2$, the numerically calculated minimal value of the mass is 3.623 at $\gamma=0.628$.

In table (I) the relative errors of the numerically calculated expressions $m_{\Sigma}$ and $m_{\text {res }} / m$ and the choices of $N$ are listed along with other results for some values of $\gamma$.

As the coordinate range of $\bar{z}$ approaches the entire real line for $\gamma \rightarrow 0$, the cosine series (27) has to be replaced by a cosine integral that can be written down explicitly as is discussed in Appendix (A), accordingly the cutoff parameter $N$ of the Fourier series has to be increased for $\gamma$ decreasing to keep the accuracy approximately constant. For practical reasons only few numerical computations were done for $\gamma<0.15$, so that the region $0<\gamma<0.15$ is left out of the figures displayed.

\section{INSTABILITY OF THE MINIMAL TORI}

An interesting question concerning surfaces of minimal area is whether they are stable - that is true minimal surfaces. Stability of a minimal surface $\Sigma$ means that for all possible one-parameter (normal) variations $\Sigma_{t}$ of the surface $\Sigma=\Sigma_{0}$ the second derivative of the area satisfies $A^{\prime \prime}(0) \geq 0$. A normal variation can be written as $\phi n^{a}$ where $n^{a}$ is a smooth unit normal vector field along $\Sigma_{0}$ and $\phi$ is a smooth function on $\Sigma$. The second variation of the area is then given by

$$
A^{\prime \prime}(0)=-\int_{\Sigma} \phi\left[\triangle_{\Sigma} \phi+\left(\tilde{R}_{a b} n^{a} n^{b}+\tilde{p}_{a b} \tilde{p}^{a b}\right) \phi\right] d A
$$

where $\Sigma$ is now taken to be our minimal toroidal boundary surface specified by $\bar{r}=1$ in the physical geometry, $\triangle_{\Sigma}$ is the Laplacian on $\Sigma, \tilde{R}_{a b}$ the Ricci curvature of $\tilde{g}$ on $\Sigma$, and $\tilde{p}_{a b}$ the extrinsic curvature of $\Sigma$. Stability holds iff all eigenvalues of the operator $-\left(\triangle_{\Sigma}+\left(\tilde{R}_{a b} n^{a} n^{b}+\tilde{p}_{a b} \tilde{p}^{a b}\right)\right)$ are nonnegative for a minimal surface. If $\Sigma$ is of toroidal topology it is known [1] that it can only be stable if the minimal torus is totally geodesic, which it cannot be in our case as was concluded from the form of $p_{a b}$ given in Eq. (14). Nevertheless it is interesting to compute the spectrum (or at least the first few eigenvalues, since this has to be done numerically). Using the Gauss law and the constraint conditions $\tilde{R}=\tilde{p}=0$, and denoting the scalar curvature of $\Sigma$ as ${ }^{2} \tilde{R}$ the eigenvalue problem becomes

$$
-\triangle_{\Sigma} \phi+\frac{1}{2}\left({ }^{2} \tilde{R}-\tilde{p}_{a b} \tilde{p}^{a b}\right) \phi=\nu \phi .
$$

Straightforward computation yields

$$
\begin{aligned}
\triangle_{\Sigma} & =G^{-4}\left(\frac{1}{1-\gamma^{2}} \frac{\partial^{2}}{\partial \bar{z}^{2}}+\frac{\partial^{2}}{\partial \varphi^{2}}\right) \\
{ }^{2} \tilde{R} & =\frac{4 G^{-4}}{1-\gamma^{2}}\left(G^{-2} G, \bar{z}^{2}-G^{-1} G, \bar{z} \bar{z}\right) \\
\tilde{p}_{a b} \tilde{p}^{a b} & =G^{-4}\left(p_{a b} p^{a b}-\frac{p^{2}}{2}\right)=\frac{1}{2} G^{-4}\left(1-\gamma^{-2}\right)^{-1} .
\end{aligned}
$$

With some rearrangement of terms the eigenvalue equation becomes

$$
\begin{gathered}
-\frac{\partial^{2} \phi}{\partial \bar{z}^{2}}-\left(1-\gamma^{2}\right) \frac{\partial^{2} \phi}{\partial \varphi^{2}}+V \phi=\nu G^{4}\left(1-\gamma^{2}\right) \phi, \\
V=-\frac{1}{4}+2\left(G^{-2} G, \bar{z}^{2}-G^{-1} G, \bar{z} \bar{z}\right),
\end{gathered}
$$

which has to be solved with periodic boundary conditions in $\bar{z}$ and $\varphi$. Since the geometry is axially symmetric, so is $G$, and the equation separates. The eigenfunctions can be written as

$$
\phi(\bar{z}, \varphi)=u_{n \nu}(\bar{z}) v_{n}(\varphi)
$$

where $v_{n}(\varphi)=\sin n(\varphi+\alpha)$ are the axial eigenfunctions degenerate in the axial phase shift $\alpha$ and for every $n=$ $0,1,2, \ldots$ one gets an equation for the $u_{n \nu}(\bar{z})$ :

$$
-u_{n \nu}^{\prime \prime}+\left(\left(1-\gamma^{2}\right) n^{2}+V\right) u_{n \nu}=\nu_{n} G^{4}\left(1-\gamma^{2}\right) u_{n \nu},
$$

which is a regular self-adjoint Sturm-Liouville problem with periodic boundary conditions for $\gamma \neq 0, \gamma_{0}$. The spectrum is thus purely discrete and bounded below (see e.g. Ref. [17], Sec. 1.4).

As a result of using periodic boundary conditions the eigenfunctions are grouped in pairs by their number of 
zeros (see e.g. Theorem 1.4.2 of Ref. [17]): The eigenfunctions $u_{k}, \mathrm{k}=0,1,2 \ldots$, with eigenvalue $\nu_{k}$ have $k$ zeros for $k$ even, and $k+1$ zeros for $k$ odd, that is $u_{1}$ has no zero, $u_{2}$ and $u_{3}$ both have two zeros, $u_{4}$ and $u_{5}$ four, etc., where the endpoints of the interval are identified.

For practical purposes it is convenient to convert the periodic boundary conditions to unmixed boundary conditions, which is made possible by noting that for Eq.(32) it is sufficient to consider only eigenfunctions that are with respect to $\bar{z}=0$. If a periodic solution $u$ exists that is neither symmetric nor antisymmetric, then the whole solution space is spanned by the linear combinations $u(x) \pm u(-x)$ and the general solution is periodic, since with $u(x)$ being a periodic solution, so is $u(-x)$. The eigenvalue is of multiplicity two in these cases, which show up in figures (3, (4) as the crossing points of branches associated with symmetric and antisymmetric eigenfunctions that have both the same number of zeros.

The problem of finding solutions with periodic boundary conditions can thus be split into the easier problems of finding all periodic symmetric and antisymmetric solutions, equivalent to imposing the unmixed boundary conditions

$$
\begin{aligned}
u^{\prime}(0) & =u^{\prime}\left(\frac{\pi}{\gamma}\right)=0 \quad \text { for symmetric solutions, } \\
u(0) & =u\left(\frac{\pi}{\gamma}\right)=0 \quad \text { for antisymmetric solutions }
\end{aligned}
$$

so that standard methods for Sturm-Liouville problems can be applied. Here routine d02kef from the NAG Fortran Library 15] was chosen to calculate eigenvalues and eigenfunctions, as was done already for Eq. (25).

The results are given in figures (3, (1) for $n=0,1$. For all values of $\gamma$ one can find at least one negative eigenvalue, demonstrating that the tori are not stable, and are thus only saddle points of the area functional. In particular the lowest eigenvalue for $n=0$ is found to be negative for all $\gamma$, and all other eigenvalues for $n=0$ become negative as well for $\gamma$ small enough, see Fig. (3). For $n=1$ only the lowest eigenvalue is negative for $\gamma>0.71$, see Fig. (何. For $n=2$ the situation is similar: again only the lowest eigenvalue is negative, but in the much smaller region $\gamma>0.97$. For $n>2$ the situation is not totally clear: if negative eigenvalues appear, they have to occur very close to $\gamma_{0}$. By explicit computation of the area of neighboring tori with $\bar{r}=$ const., it was found that the minimal tori have minimal area for $\gamma<0.891$, and maximal area for $\gamma>0.891$ among neighboring tori of constant $\bar{r}$.

In the limiting case $\gamma=\gamma_{0}$ the Green function $G$ diverges, and all eigenvalues $\nu$ go to zero. In the other limiting case, $\gamma=0$, the coordinate range is the entire real line, and the Sturm-Liouville problem becomes singular. The potential $V$ falls off exponentially to the value $n^{2}-1 / 4$ for large $|\bar{z}|$ - note that this value is negative for $n=0$ and positive for $n>0$. While nothing special happens to the part of the spectrum with $n>0$, see
Fig. (4) for $n=1$, the situation is different for $n=0$. The general solution $u$ to Eq. (32) for $\gamma=n=0$ and arbitrary $\nu$ is asymptotically trigonometric (see e.g. Ref. [18], section 5.11), that is

$$
u=\alpha \sin (\bar{z}+\beta)+O(1 / \bar{z}),
$$

which is oscillatory with finite amplitude near $\bar{z}= \pm \infty$. Periodic boundary conditions thus can not be imposed on these solutions, for small $\gamma$ all eigenvalues decrease unboundedly, marking a growing instability of the minimal surface with respect to axially symmetric deformations.

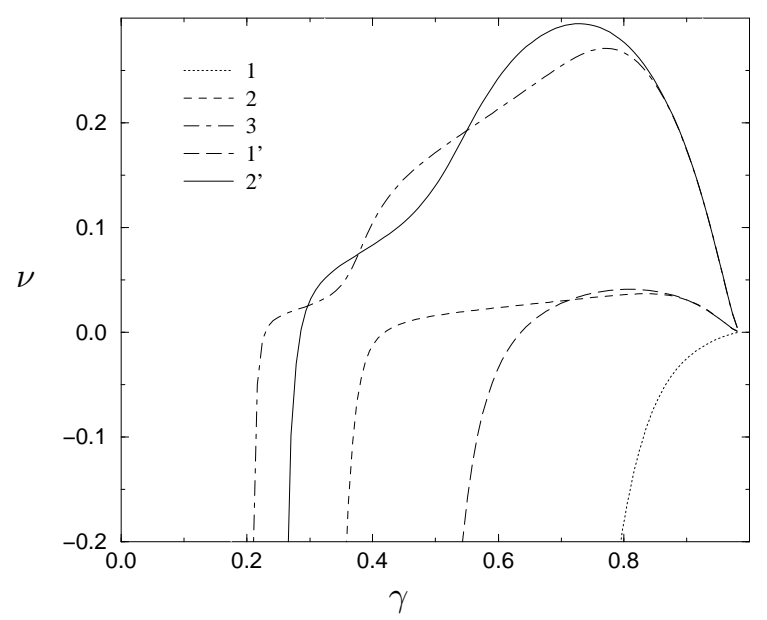

FIG. 3. The first five eigenvalues $\nu_{0 l}(n=0)$ are plotted as functions of $\gamma$ from top down $(l=1, \ldots, 5)$. The curves labelled 1,2,3 are the first three branches of eigenvalues with symmetric eigenfunctions, while $1^{\prime}, 2^{\prime}$ label the first two branches of eigenvalues with antisymmetric eigenfunctions.

\section{APPARENT HORIZONS}

As was shown before, the minimal tori considered here are all unstable, so that they locally can be deformed to an outer trapped surface [1]. By asymptotic flatness the region of compact outer trapped surfaces has to be finite, the outer boundary is called apparent horizon, which is a stable marginally outer trapped surface (see Ref. [1] and references cited therein). Noting that $\tilde{g}$ is analytic by construction of the conformal factor as the solution of Eqs. (28 30) and not flat (by analyticity it would have to be flat everywhere if it were flat in some region, but this can not be, since the mass is positive by Eq. (31)), it can be concluded from Ref. [1] that the apparent horizon has spherical topology.

The existence and properties of apparent horizons have received some interest in the context of the cosmic censorship hypothesis 19, where they play a double role as 


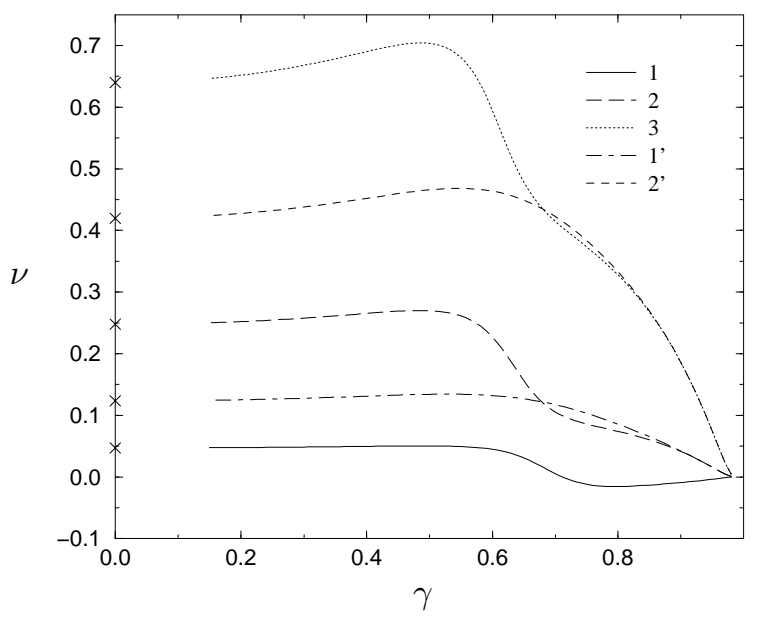

FIG. 4. The first five eigenvalues $\nu_{1 l}(n=1)$ are plotted as functions of $\gamma$ from top down $(l=1, \ldots, 5)$, the values for $\gamma=0$ are marked by $\times$ 's. The curves labelled $1,2,3$ are the first three branches of eigenvalues with symmetric eigenfunctions, while $1^{\prime}, 2^{\prime}$ label the first two branches of eigenvalues with antisymmetric eigenfunctions.

in Penrose's singularity theorem 10 and also as a local (albeit only weak) analogue of the global concept of an event horizon. The second is practically exploited in the apparent horizon boundary condition [5] used in numerical relativity and also was used in a new approach for the definition of a black hole itself 20 .

The apparent horizons were located numerically and analyzed with respect to the Penrose and hoop conjectures. Penrose [19] formulated the conjecture that if a collapsing body satisfies the inequality

$$
m<\sqrt{\frac{A}{16 \pi}}
$$

where $m$ is the ADM mass and $A$ the area of a trapped surface, then cosmic censorship should be violated. As pointed out by Horowitz [21], if $A$ is the minimal area that surrounds a trapped surface (in the time symmetric case identical to the apparent horizon) and satisfies the above inequality, then cosmic censorship does not hold. For the apparent horizons found here the inequality is always violated, the Penrose conjecture thus confirmed, see figure (5).

The hoop conjecture, due to Thorne [11], states that an apparent horizon will exist if and only if a mass $m$ gets compacted into a region such that the circumference $C$ satisfies $C \leq 4 \pi m$ in every direction. The exact definition of what should be meant by $m$ and $C$ is left open. For a precise definition of $m$ one would need a notion of quasilocal mass which is available only for the spherically symmetric case (no gravitational waves), here the ADM mass was chosen. Also for a general definition of the circumference various definitions would be possible. In an axially symmetric geometry which is also symmetric with respect to reflection at the equator, straightforward definitions of circumference exist along the equator $\left(C_{e}\right)$ and along a meridian circle $\left(C_{p}\right)$ :

$$
\begin{aligned}
C_{e} & =\left.2 \pi \psi^{2} R\right|_{\theta=\pi / 2}, \\
C_{p} & =4 \int_{0}^{\pi / 2} d \theta \psi^{2} \sqrt{R^{\prime 2}+R^{2}},
\end{aligned}
$$

where $R$ is the radial coordinate in standard polar coordinates $(R, \theta, \varphi)$. The results for $\frac{C}{4 \pi m}$ are given in Fig. (6) and table (I).

For the problem of locating apparent horizons in the axially symmetric case a variety of different methods is available by now. If one considers configurations which are symmetric (or almost symmetric) with respect to reflection at the equator, one can reasonably hope that the apparent horizon will be expressible as a function $R(\theta)$. This gives the ODE

$$
\frac{R^{\prime \prime}-\frac{R^{\prime 2}}{R}}{1+\frac{R^{\prime 2}}{R^{2}}}-4 R^{2} \frac{\psi_{R}}{\psi}+R^{\prime}\left(4 \frac{\psi_{\theta}}{\psi}+\cot \theta\right)-2 R=0,
$$

which has to be solved with the boundary conditions

$$
R^{\prime}(0)=R^{\prime}(\pi / 2)=0 .
$$

This is done numerically using a shooting and matching method (NAG 15] routines d02agf and d02ebf were used in two different codes for horizon finding).

The results are shown in figures (5), (6) and table (I). For $\gamma \rightarrow \gamma_{0}$ the apparent horizon becomes spherical and pinches off to infinity as has been discussed for the analogous case when the Yamabe number goes to zero for a manifold without boundary in Ref. [3]. For $\gamma<\gamma_{0}$ the apparent horizon is oblate, but not very strongly.

\section{DISCUSSION}

In a previous paper [12] the notion of toroidal conformal symmetry was introduced to label a class of initial data where a $U(1) \times U(1)$ conformal isometry is present. Working on a compactified background metric allows to easily exploit this conformal symmetry to decompose the Lichnerowicz conformal factor in a double Fourier series on the group orbits and give the solution in terms of a countable family of uncoupled ODEs on the orbit space. The present paper applies these methods to construct and analyze initial data containing a marginally outer trapped torus. Special emphasis is given to the investigation of the limiting cases when the sequence of solutions intersects the boundary of conformal superspace.

To get a better understanding of the results it seems useful to look at the usually studied case of vacuum asymptotically flat data without boundary, where the 


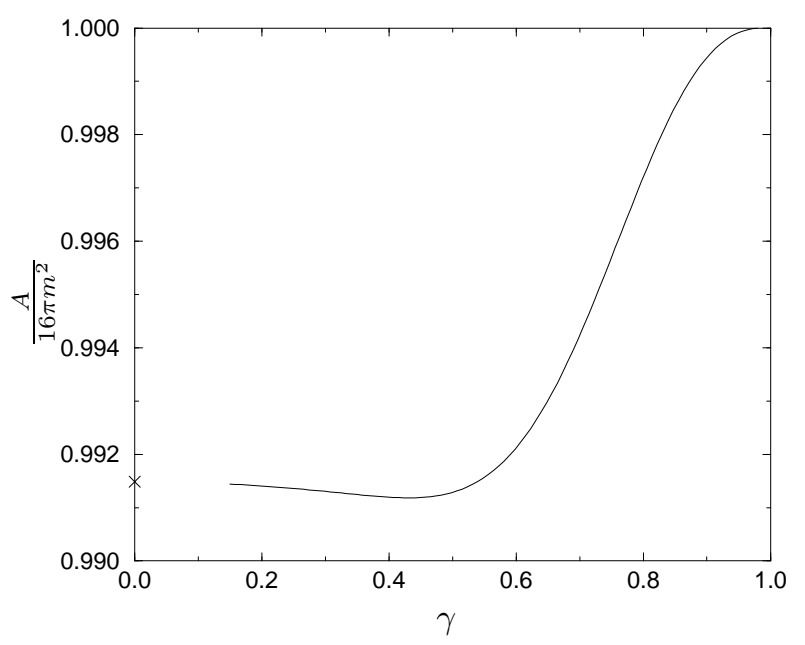

FIG. 5. Check of the Penrose isoperimetric inequality, the value for $\gamma=0$ is marked by $\times$.

cases understood best are the time-symmetric, notably the axially symmetric subclass known as Brill-waves [22.

In order to simplify this comparison I will first discuss generalizations of the data considered in this paper. The obvious generalization is to drop conformal flatness and consider deformations of the base metric $g$ of the form

$$
g=e^{2 A q(\bar{r})}\left(\frac{d \bar{r}^{2}}{1-\gamma^{2} \bar{r}^{2}}+\left(1-\gamma^{2} \bar{r}^{2}\right) d \bar{z}^{2}\right)+\bar{r}^{2} d \varphi^{2},
$$

which is the analogue of the Brill-wave ansatz [4] while keeping the conformal symmetry, and is currently investigated. The general axially symmetric case corresponds to form functions $q(\bar{r}, \bar{z})$ that depend on both $\bar{r}$ and $\bar{z}$. Within the present ansatz one could consider certain nonaxially symmetric situations by placing $\Lambda$ off the axes (the sets of fixed points of the isometries), the physical metric then has no isometries whatsoever - since an isometry of $g$ carries over to $\tilde{g}$ only if $\Lambda$ is a fixed point of this isometry [23].

The usually studied case is recovered, finally, by imposing regularity on all of $R^{3}$ instead of the $\tilde{p}=0$ boundary condition, this case has been discussed in 12], the publication of numerical results is in preparation.

For these traditionally considered data sequences of metrics which approach the boundary of the space of initial data have been studied both analytically and numerically and tested for apparent horizons. Two complementary paths have been taken, an overview is given in 22]. Sequences of metrics for which the Yamabe number goes to zero have been studied analytically by Beig and Ó Murchadha [3], who proved that asymptotically the mass diverges in indirect proportion to the Yamabe number and that an apparent horizon would always appear before the mass diverges. Abrahams et al. [24] on

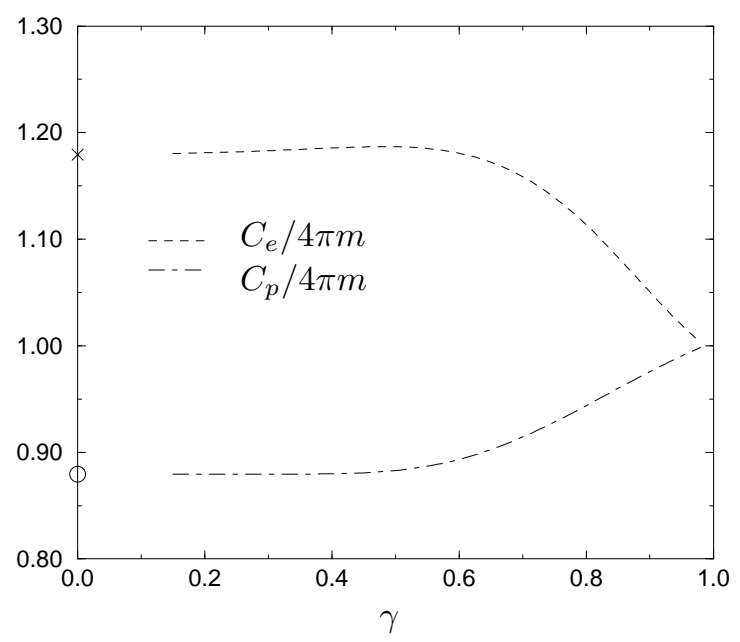

FIG. 6. Equatorial and polar hoop quotients, the values for $\gamma=0$ are marked by $\times$ and $\circ$.

the other hand studied families of metrics of fixed mass with a singular limit for which no apparent horizon appeared even in the limiting case.

The limiting case $\gamma \rightarrow \gamma_{0}$ of the present paper is similar to the one where the Yamabe number goes to zero. As the Sobolev quotient defined in Eq. (11) is just an extension of the Yamabe number including an additional boundary term, the mathematical and physical situation is analogous. As the mass diverges, the apparent horizon becomes more and more spherical and pinches off to infinity. Going beyond that critical point the mass becomes infinitely negative. Whereas in the no-boundary case the amplitude parameter $A$ of Eq. (33) can be increased without bound, which gives rise to an infinite number of analogous singularities in the mass function (to be discussed in forthcoming work), here the parameter $\gamma$ is bounded from above by $\gamma \leq 1$, and the mass appears to stay negative for $\gamma>\gamma_{0}$. A proof that the Sobolev quotient will become negative was unsuccessful due to the finite range of $\gamma$ : In the Brill-wave case an estimate can be obtained with simple test-functions by just increasing the amplitude enough [22] - here the same trick can not be carried out. $\gamma_{0}$ comes in fact very close to the upper boundary $\gamma=1$, and so a very good choice of test function would have to be made.

The other case where the sequence of solutions hits the boundary of conformal superspace, $\gamma \rightarrow 0$, is fundamentally different. While in the first case the base geometry stays regular, the situation when the boundary torus touches the axis can be considered as singular, nevertheless the mass and location of the apparent horizon remain finite, but a curvature singularity develops at the origin.

Table (I) lists some numerical results for different values of $\gamma$. 


\begin{tabular}{c|c|c|c|c|c|c}
\hline \hline$\gamma$ & $m$ & $\lambda_{1}$ & $C_{p} / C_{e}$ & $m_{\text {res }} / m$ & $\frac{m_{\Sigma}}{\pi / 2}-1$ & $N_{\max }$ \\
\hline 0.0 & 3.8771 & 0.4700 & 0.4758 & - & - & - \\
\hline 0.2 & 3.8442 & 0.4639 & 0.7447 & $3.7 \times 10^{-10}$ & $-3.7 \times 10^{-6}$ & 100 \\
\hline 0.4 & 3.7444 & 0.4443 & 0.7421 & $1.6 \times 10^{-9}$ & $6.4 \times 10^{-7}$ & 37 \\
\hline 0.5 & 3.6771 & 0.4283 & 0.7440 & $2.1 \times 10^{-9}$ & $-7.0 \times 10^{-9}$ & 33 \\
\hline 0.6 & 3.6272 & 0.4068 & 0.7567 & $4.1 \times 10^{-9}$ & $-3.3 \times 10^{-8}$ & 24 \\
\hline 0.7 & 3.6571 & 0.3775 & 0.7891 & $2.5 \times 10^{-8}$ & $-3.9 \times 10^{-8}$ & 19 \\
\hline 0.8 & 3.9173 & 0.3353 & 0.8476 & $7.6 \times 10^{-8}$ & $-1.1 \times 10^{-7}$ & 15 \\
\hline 0.9 & 5.0129 & 0.2636 & 0.9287 & $2.5 \times 10^{-7}$ & $-1.1 \times 10^{-7}$ & 12 \\
\hline 0.989 & 226.13 & 0.0773 & 0.9998 & $4.8 \times 10^{-4}$ & $7.8 \times 10^{-7}$ & 7 \\
\hline \hline
\end{tabular}

TABLE I. Results from numerical calculations, numbers are rounded to the last digit.

The technique of using conformal symmetry together with compactification had several technical advantages. First of all, the ODEs (28) and (25) resulting from the Hamiltonian constraint and existence question can be handled with relatively small numerical expertise and desktop workstations. The use of the NAG numerical Fortran library 15] yields an excellent documentation of all numerical routines that have been used.

Another point in favor of the method is that it works well for strong data - that is near $\gamma=\gamma_{0}$ in our sequence, only the $n=0$ term in the Fourier series blows up when the mass does.

If there are additional asymptotic regions (representing black holes), discrete symmetries result from an arrangement compatible to the conformal isometry as discussed in [12].

It is hoped, that the technical approach taken here and in [12], namely to use compactification and conformal Killing vector fields may also prove useful in other situations.

\section{ACKNOWLEDGMENTS}

The author thanks R. Beig for his support, encouragement and for many stimulating discussions. This work was financially supported by Fonds zur Förderung der wissenschaftlichen Forschung, Project No. P09376-PHY.

\section{APPENDIX A: THE EXPLICIT LIMITING SOLUTION}

In the limiting situation $\gamma=0$ the constraint equation (22) can be solved explicitly. Making the ansatz

$$
G=\frac{\sqrt{2}}{\sqrt{\bar{r}^{2}+\bar{z}^{2}}}+\phi
$$

(analogous to (26)), we get

$$
\begin{aligned}
\triangle \phi & =0, \\
\left.\left(\frac{\partial \phi}{\partial \bar{r}}+\frac{1}{4} \phi\right)\right|_{\bar{r}=1} & =\left(1+\bar{z}^{2}\right)^{-3 / 2}-\frac{1}{4}\left(1+\bar{z}^{2}\right)^{-1 / 2}
\end{aligned}
$$

from Eqs. (22) and (23).

We solve by cosine transformation:

$$
\phi(\bar{r}, \bar{z})=\frac{2}{\pi} \int_{0}^{\infty} \cos (\omega \bar{z}) f_{\omega}(\bar{r}) d \omega,
$$

resulting in a family of Bessel equations for regular functions $f_{\omega}(\bar{r})$ parameterized by $\omega$ :

$$
f_{\omega}^{\prime \prime}+\frac{1}{r} f_{\omega}^{\prime}-\omega^{2} f_{\omega}=0 .
$$

The regular solutions are

$$
f_{\omega}(\bar{r})=c(\omega) I_{0}(\omega \bar{r}),
$$

where $I_{n}$ and $K_{n}$ used below are modified Bessel functions of order $n$ and $c(\omega)$ is determined from the cosine transformed boundary condition

$$
\begin{aligned}
f_{\omega}^{\prime}(\bar{r})+\frac{1}{4} f_{\omega}(\bar{r}) & =\sqrt{\frac{2}{\pi}} \int_{0}^{\infty} \cos (\omega \bar{z}) \\
& \times\left(\left(1+\bar{z}^{2}\right)^{-3 / 2}-\frac{1}{4}\left(1+\bar{z}^{2}\right)^{-1 / 2}\right) d \omega \\
& =\frac{1}{4} \sqrt{\frac{2}{\pi}}\left(4 \omega K_{1}(\omega)-K_{0}(\omega)\right) .
\end{aligned}
$$

as

$$
c(\omega)=\sqrt{\frac{2}{\pi}} \frac{4 \omega K_{1}(\omega)-K_{0}(\omega)}{4 \omega I_{1}(\omega)+I_{0}(\omega)} .
$$

We get an explicit formula for the Green function,

$$
\begin{aligned}
G(\bar{r}, \bar{z}) & =\frac{\sqrt{2}}{\sqrt{\bar{r}^{2}+\bar{z}^{2}}}+\phi=\frac{\sqrt{2}}{\sqrt{\bar{r}^{2}+\bar{z}^{2}}} \\
& +\frac{2}{\pi} \int_{0}^{\infty} \cos (\omega \bar{z}) \frac{4 \omega\left(K_{1}(\omega)-K_{0}(\omega)\right.}{4 \omega I_{1}(\omega)+I_{0}(\omega)} I_{0}(\omega \bar{r}) d \omega .
\end{aligned}
$$

$\phi$ is a regular function of $\bar{r}, \bar{z}$, the integrand diverges only logarithmically for small $\omega$ and exhibits exponential falloff for large $|\bar{z}|$. Its value at the point at infinity gives the ADM mass $m=3.877$ by $m=2 \sqrt{2} \phi(\Lambda)$.

Alternatively the Green function can be written as

$$
\begin{aligned}
G(\bar{r}, \bar{z})= & \frac{2}{\pi} \int_{0}^{\infty} \cos (\omega \bar{z}) \frac{p(\omega, \bar{r})}{q(\omega)} d \omega, \\
p(\omega, \bar{r})= & 4 \omega\left(I_{1}(\omega) K_{0}(\omega \bar{r})+K_{1}(\omega) I_{0}(\omega \bar{r})\right) \\
& +K_{0}(\omega \bar{r}) I_{0}(\omega)-I_{0}(\omega \bar{r}) K_{0}(\omega), \\
q(\omega)= & 4 \omega I_{1}(\omega)+I_{0}(\omega) .
\end{aligned}
$$

using the identity 25]

$$
\frac{1}{\sqrt{\bar{r}^{2}+\bar{z}^{2}}}=\frac{2}{\pi} \int_{0}^{\infty} \cos (\omega \bar{z}) K_{0}(\omega \bar{r}) d \omega .
$$


Positivity of $G(\bar{r}, \bar{z})$ was checked by numerical evaluation, the behavior of $G$ for large $|\bar{z}|$ can be seen analytically by integration of (A2) in the complex plane. Both $p$ and $q$ are positive and symmetric on the real line, but $q$ has infinitely many zeros for purely imaginary arguments, leading to corresponding poles of $p / q$. Observing, that the factor $\exp (-\operatorname{Im}(\omega) \bar{z})$ makes contributions to the integral small for large $\bar{z}$ in the upper complex half plane (for $\bar{z} \rightarrow-\infty$ the lower half respectively) yields an asymptotic formula for the integral by choosing a path that encircles only the first pole (an asymptotic series results from summing over all poles),

$$
G(\bar{r}, \bar{z})=c(\bar{r}) \exp (-\alpha|\bar{z}|)
$$

where $\alpha=0.6856$ is the first zero of $q(\omega)$ on the imaginary axis and

$$
c(\bar{r})=4 i \text { Residual }\left(\frac{p(\omega, \bar{r})}{q(\omega)}, \omega=0.6856\right) .
$$

[1] G. J. Galloway, in Differential Geometry and Mathematical Physics, Contemporary Mathematics, Vol. 170, eds. J. Beem and K. Duggal (American Mathematical Society, Providence, 1994), p. 113.

[2] See e.g. Y. Choquet-Bruhat and J. W. York, Jr., in General Relativity and Gravitation, ed. A. Held (Plenum, New York, 1980) Vol. 1.

[3] See e.g. R. Beig and N. Ó Murchadha, Phys. Rev. Lett. 66, 2421 (1991) and references cited therein.

[4] D. R. Brill, Ann. Phys. (N.Y.) 7, 466 (1959).

[5] E. Seidel and W.-M. Suen, Phys. Rev. Lett. 69, 1845 (1992).

[6] See Ref. 8] and the account of the Yamabe problem and its connection to General Relativity given in N. Ó Murchadha, in Proceedings of the Centre for Mathematical Analysis, edited by R. Bartnik (Australian National University, Canberra, 1989), Vol. 19, p. 137.

[7] J. F. Escobar, J. Diff. Geom. 35, 21 - 84 (1992).

[8] Lee and Parker, Bull. Am. Math. Soc. 17, 37 (1987).

[9] R. Schoen and S. T. Yau, Comm. Math. Phys. 65, $45-$ 76 (1979).

[10] R. Penrose, Phys. Rev. Lett. 14, 57 (1965).

[11] K. S. Thorne, in Magic Without Magic: John Archibald Wheeler, edited by J. Klauder (Freeman, San Francisco, 1972), p 231.

[12] R. Beig and S. Husa, Phys. Rev. D 50, 12 (1994).

[13] For an overview on the positive mass theorem of Schoen and Yau [9] and its relation to the Yamabe problem see Ref. [8].

[14] See e.g. J. L. Kazdan, in College on Differential Geometry, Summer School Report, (ICTP, Trieste, 1989).

[15] NAG FORTRAN Library Manual - Mark 16, The Numerical Algorithms Group Ltd., Oxford (1994).
Some information is also available over WWW, at http://www.nag.co.uk/.

[16] D. Bernstein, D. Hobill, E. Seidel and L. Smarr, Phys. Rev. D 15, 50, 3760 (1994).

[17] B. M. Levitan and I. S. Sargsjan, Introduction to Spectral Theory, Translations of Mathematical Monographs, Vol. 38 (American Mathematical Society, Providence, 1975).

[18] R. Courant and D. Hilbert, Methods of Mathematical Physics, Vol. 1 (John Wiley-Interscience, New York, 1953).

[19] R. Penrose, Rivista del Nuovo Cimento 1, 251 (1969), Ann. NY Acad SCi 224, 125 (1973)

[20] S. A. Hayward, Phys. Rev. D 49, 6467 (1994).

[21] G. Horowitz, in Lecture notes in Physics 202, edited by F. J. Flaherty (Berlin, Springer, 1984).

[22] N. Ó Murchadha, in Directions in General Relativity, Vol. 2, edited by B. L. Hu and T. A. Jacobson (C.U.P., Cambridge, 1993).

[23] R. Beig, Class. Quant. Grav. 8, L205 (1991).

[24] A. M. Abrahams, K. R. Heiderich, S. L. Shapiro and S. A. Teukolsky, Phys. Rev D 46, 24452 (1992).

[25] I. S. Gradshteyn, Table of Integrals, Series and Products (Academic Press, New York, 1980). 